\title{
Abandonner le théâtre pour la politique...
}

\section{Michel Biard}

\section{(2) OpenEdition \\ Journals}

Édition électronique

URL : https://journals.openedition.org/ahrf/5453

DOI : 10.4000/ahrf.5453

ISSN : 1952-403X

\section{Éditeur :}

Armand Colin, Société des études robespierristes

\section{Édition imprimée}

Date de publication : 1 juin 2003

Pagination : 171-176

ISSN : 0003-4436

\section{Référence électronique}

Michel Biard, «Abandonner le théâtre pour la politique... », Annales historiques de la Révolution française [En ligne], 332 I avril-juin 2003, mis en ligne le 22 avril 2008, consulté le 24 avril 2022. URL : http:// journals.openedition.org/ahrf/5453 ; DOI : https://doi.org/10.4000/ahrf.5453 


\section{SOURCES}

\section{ABANDONNER LE THÉÂTRE POUR LA POLITIQUE...}

Le 17 mars 1791, Collot d'Herbois passait avec les administrateurs du Théâtre de Monsieur, Autié et Viotti, un contrat par lequel il s'engageait à collaborer avec ce Spectacle pour une durée de quatre ans (1). Ce contrat prenant effet rétroactif à dater de janvier 1791, Collot d'Herbois était ainsi susceptible de livrer ses œuvres au Théâtre de Monsieur jusqu'à la fin de 1794. Le contrat fixait la rétribution accordée à l'auteur, soit 30 livres par acte pour les dix premières représentations, données impérativement en l'espace de deux mois; 24 livres par acte pour les dix suivantes, à donner dans les quatre mois après la dixième représentation; 18 livres par acte pour les dix suivantes, à placer dans le programme des huit mois suivant la vingtième représentation; enfin encore 18 livres par acte pour toute représentation ultérieure, la pièce devant être jouée au moins dix fois au cours d'une saison théâtrale entière, à l'issue des quatorze premiers mois de représentation. En cas d'insuccès, les directeurs du Théâtre étaient libres de modifier l'accord et de ne donner la pièce que quinze fois dans un délai de six mois (au lieu de vingt fois), à la rétribution maximale, sous réserve de prévenir l'auteur avant la sixième représentation. Le délai écoulé, l'œuvre redevenait propriété pleine et entière de l'auteur, à la différence de ce qui se passait avant la Révolution lorsque le Théâtre Français s'emparait des pièces « tombées dans les règles ", c'est-à-dire n'ayant pas eu de succès.

(1) Michel BIARD, "Jean-Marie Collot d'Herbois. Homme de theâtre et homme de pouvoir (1749-1796) ", thèse de doctorat dactylo., sous la direction de $M$. Vovelle, Université Paris I PanthéonSorbonne, 1993, tome I, pp. 200-202. Cf. également la version publiée de ce travail sous le titre Collot d'Herbois. Légendes noires et Révolution, Lyon, Presses Universitaires de Lyon, 1995, pp. 74-75. 
Avant la signature de ce contrat, Collot d'Herbois avait déjà donné au Théâtre de Monsieur deux pièces : La Famille patriote ou la Fédération, "pièce nationale en deux actes et en prose, suivie d'un divertissement", jouée pour la première fois le 16 juillet 1790 (2); puis Le Procès de Socrate, ou le Régime des anciens temps, comédie en trois actes et en prose, représentée à partir du 9 novembre 1790. La première, quoique simple cuvre de circonstance, remporta un succès non négligeable avec seize représentations en moins d'un mois, avant d'obtenir une présence tout à fait rare dans le répertoire des mois suivants. Après quelques reprises, elle fut notamment donnée par le Théâtre de Monsieur le 15 juillet 1791, dans un contexte qui avait bien évolué depuis la fuite du roi avortée à Varennes. Pour sa part, $L e$ Procès de Socrate connut douze représentations lors du premier mois avant de quasiment disparaître. Il est vrai que la pièce avait profondément divisé les critiques sur des questions ouvertement politiques.

Au moment où, en mars 1791, Collot d'Herbois signe son contrat avec le Théâtre de Monsieur, Le Procès de Socrate a connu deux reprises éphémères en février. Le Théâtre vient de s'installer dans sa nouvelle salle de la rue Feydeau (le 6 janvier), ce qui occasionne sans doute la rédaction du contrat. Mais plus encore que ce changement de salle, c'est le succès d'une autre œuvre qui conduit sans doute les deux parties à négocier. La première pièce de Collot d'Herbois en 1791, Les Portefeuilles, comédie en deux actes et en prose, est en effet jouée le 10 février. Elle obtient aussitôt un vif succès : quinze représentations en un mois, puis quatre à cinq représentations dans les mois suivants jusqu'en décembre (sauf en juillet et août), soit au total une bonne cinquantaine de représentations. Ajoutons que la Veuve Duchesne réédite la comédie en 1791, là où une seule édition avait été faite des pièces précédentes, et que, de plus, elle est également publiée en province (au moins à Avignon en 1791, puis en 1792, mais aussi à Nyons en l'an III). Avec les clauses du contrat signé le 17 mars 1791 et donc valables depuis janvier afin de prendre en compte rétroactivement Les Portefeuilles, cette comédie doit rapporter à son auteur un peu plus de 2300 livres, soit une somme confortable (sur des bases similaires, La Famille patriote aurait rapporté deux fois moins). Tout semble attester que Collot d'Herbois est en ces premiers mois de 1791 un auteur comblé, d'autant que la presse est presque unanime dans les louanges vis-à-vis des Portefeuilles. Même Les Sabats jacobites, peu tendres avec les membres du club des Jacobins, constatent : "Cette pièce [...] a obtenu un succès mérité [...] La représentation de cette petite comédie n'a rien laissé à désirer » (3). Une partie de ce succès tient au fait que l'auteur a assez largement délaissé les allusions politiques, contrairement à ce qu'il avait fait dans Le Procès de

(2) Et non le 17 juillet comme l'indique l'édition parue à Paris chez la Veuve Duchesne.

(3) $\mathrm{N}^{\circ} 2,1791$. 
Socrate. Le triomphe est tel que, lors d'une représentation donnée le 20 septembre 1791 pour célébrer les vertus de la Constitution enfin adoptée, on peut noter un engouement toujours vif : «Le peuple s'y est porté avec une telle affluence, que l'on comptait dans les premières loges, qui contiennent six personnes, jusqu'à douze ou treize spectateurs. Tout, jusqu'aux corridors, était plein de manière à ne pouvoir pas s'y remuer. Cette nombreuse assistance a été parfaitement tranquille » (4). Près de vingt ans après sa première pièce, que peut demander de plus un auteur qui a mis sa plume au service des idées nouvelles? A priori, tout semblerait devoir le pousser à récidiver, et pourtant il faut attendre environ onze mois avant qu'il ne crée une nouvelle œuvre.

Cette année 1791 est en fait pour Collot d'Herbois un temps d'engagement politique accru. À la fin du printemps, il tend à se spécialiser dans la défense des soldats «opprimés » par leurs officiers et prend notamment la parole au club des Jacobins à de multiples reprises pour défendre les Suisses du régiment de Châteauvieux victimes dans l'été 1790 d'une terrible répression menée par le marquis de Bouillé. Cet engagement le pousse alors sur le devant de la scène politique et il obtient une célébrité d'ampleur nationale à la fin d'octobre 1791 en remportant le concours ouvert par les Jacobins pour la rédaction d'un almanach patriotique. Son Almanach du Père Gérard connaît en effet un immense succès et ce ne sont pas moins de trois éditions successives qui sortent à Paris en novembre 1791, avant que plusieurs nouvelles éditions paraissent en 1792, à nouveau à Paris, dans les départements (y compris en breton (5), en provençal, en flamand et en allemand) et même à Londres. L'homme semble devoir mettre de côté son amour pour le théâtre pour jouer sur la grande scène politique. Il y a dix ans, j'écrivais dans ma thèse que, avec le début de l'année 1792 et sa dernière pièce (L'Aîné et le Cadet), «il ne reprend plus la plume, sous réserve de pièces inédites qu'il a pu conserver dans ses papiers saisis en l'an III et aujourd'hui disparus » (6). De fait, l'année 1792 est pour lui plus que jamais tournée vers la politique, avec d'abord les débats sur la guerre, puis la fête en l'honneur des Suisses de Châteauvieux libérés du bagne (avril), le 10 août, enfin son élection comme représentant du peuple à Paris (au troisième rang, derrière Robespierre et Danton, ce qui atteste sa popularité). J'imaginais bien qu'il n'avait pu abandonner le théâtre sans déchirements intérieurs, or le document inédit publié dans le présent numéro des A.H.R.F. vient le démontrer.

(4) Almanach général des spectacles (...), Paris, Froullé, 1792, pp. 68-69.

(5) Cette édition en breton est devenue très rare. Au moment où j'effectuais des recherches pour ma thèse, je n'en avais pas retrouvé la trace. Il était pourtant évident, étant donné le sujet de l'almanach, qu'une édition bretonne avait dû voir le jour. J'ai depuis été contacté par un chercheur breton qui l'a localisée et s'apprête à en donner une publication bilingue (breton-français).

(6) Collot d'Herbois. Légendes (...), op. cit., p. 87. J'avais alors simplement souligné qu'il prenait encore la plume pour rédiger des critiques, notamment dans l'almanach des Spectacles de Paris, et de toute la France en 1792, 1793 et sans doute 1794 (édités chez la Veuve Duchesne). 
Ce "Traité de $\mathrm{M}$. Collot d'Herbois", généreusement mis à notre disposition par un collectionneur suisse, monsieur Claude Lebet (La Chauxde-Fonds), prouve qu'à la fin de l'année 1791 le ci-devant Théâtre de Monsieur - à présent dit "de la rue Feydeau », afin d'éviter la référence à Monsieur, frère du roi, désormais émigré - et Collot d'Herbois avaient encore nombre de projets en cours. Autié et Viotti pouvaient-ils d'ailleurs faire autrement que de relancer un homme qui venait d'obtenir le succès avec Les Portefeuilles et qui atteignait une immense notoriété avec l'Almanach du Père Gérard ? La date ne saurait être innocente. Le traité est en effet signé le 15 novembre 1791, c'est-à-dire précisément au moment où les entretiens du Père Gérard et des paysans d'Tlle-et-Vilaine font de Collot d'Herbois une plume fameuse. Les clauses sont intéressantes à plus d'un titre. L'auteur s'engage tout d'abord à donner au Théâtre de la rue Feydeau cinq œuvres en deux actes, deux comédies et trois opéras dont les sujets ne sont pas précisés, mais qui sont déjà arrêtés puisque les deux directeurs écrivent : «... les sujets nous sont connus [...] ». Chacun de ces ouvrages devra être représenté vingt fois dans les deux années qui s'écouleront à partir de Pâques 1792 au plus tard (7), c'est-à-dire pour les saisons 1792-1793 et 1793-1794, soit les deux dernières saisons incluses dans le contrat du 17 mars 1791 . Les conditions financières sont d'ailleurs celles «... exprimées dans le traité précédemment conclu [...]», qu'au demeurant Autié et Viotti reconnaissent avoir " égaré »!

Collot d'Herbois s'engage à livrer son premier manuscrit sous quinzaine. Il s'agit de toute évidence de L'Aîné et le Cadet, représentée pour la première fois au Théâtre de la rue Feydeau le 17 janvier 1792. La pièce a obtenu des critiques élogieuses dans l'ensemble, mais n'en a pas moins très vite été retirée de l'affiche. Six représentations en janvier, une reprise en février, une autre en mars, puis vient le silence. Or, le traité passé le 15 novembre 1791 précise que le premier opéra de Collot d'Herbois devait être fourni avant le 15 décembre, soit dans un délai d'un mois ( « si cela est nécessaire », précise-t-on). Pourtant, dans les dernières semaines de 1791, un seul opéra français est représenté au Théâtre de la rue Feydeau, Les Deux Nicodèmes, ou les Français dans la planète de Jupiter, " opéra folie " de Beffroy de Reigny (le 21 novembre). Le répertoire de Tissier (8) recense sept opéras français donnés à ce Théâtre en 1792, de janvier jusqu'au début d'août (9). Les autres auteurs contactés pour des opéras français et joués pendant cette période sont Demoustier, Désaugiers fils, Framery, Parisau (ou Pariseau) et Verneuil. Parmi ceux-ci, Parisau est de loin le plus prolifique. Il a déjà collaboré régulièrement avec le

(7) Rappelons qu'une saison théâtrale normale s'étend alors sur deux années civiles, car, pour des raisons religieuses, une coupure intervient lors des fêtes pascales.

(8) André Tissier, Les Spectacles à Paris pendant la Révolution (...), Genève, Droz, 1992, tome I.

(9) Les opéras français sont un genre auquel tiennent les administrateurs du Théâtre (ci-devant) de Monsieur. Dans cette même période (17 janvier-4 août 1792), quatorze œuvres nouvelles sont en effet offertes au public parisien. C'est dire que la part des opéras français représente la moitié des nouveautés, ce qui explique probablement la demande faite à Collot d'Herbois. 
Thêâtre de Monsieur et il illustre bien la position des auteurs qui créent tant des comédies que des opéras français (trois comédies et deux opéras joués en 1790 sur ce Théâtre). Demoustier est, lui, plutôt un auteur de comédie (il en a donné trois à ce Théâtre en 1790 et 1791). Desaugiers fils n'a écrit que les paroles de l'opéra français donné rue Feydeau en janvier 1792, et mis en musique par son propre père (Le Médecin malgré lui). Framery ne crée en ces premières années de Révolution que La Colonie, opéra français joué en juin 1792 ; de même pour Verneuil, avec L'Avare puni en août 1792. En promettant à Autié et Viotti deux comédies et trois opéras, Collot d'Herbois ne fait donc que suivre une voie déjà empruntée par d'autres, notamment par Parisau.

Un seul des sept opéras français alors joués au Théâtre de la rue Feydeau est resté anonyme, sans doute en raison de son échec immédiat puisqu'il n'a qu'une seule représentation, le 23 juin 1792. L'opéra Les Plaideurs, « d'après Racine ", est-il de la main de Collot d'Herbois? L'hypothèse ne peut être exclue, toutefois le fait qu'il soit en trois actes et surtout la date tardive m'inclinent plutôt à ne pas l'affirmer. Il parait plus probable que Collot d'Herbois et les directeurs du Théâtre ont rompu leurs liens beaucoup plus tôt, sans doute en raison des engagements politiques de l'auteur (10). De plus, trois autres œuvres devaient être livrées avant Pâques 1792, une seconde comédie et deux opéras. Or, à ma connaissance, aucune de ces pièces n'a été jouée.

Reste une dernière découverte occasionnée par ce document inédit. Non seulement Collot d'Herbois possédait sans doute encore dans ses papiers au moins une comédie nouvelle, mais de plus il s'était engagé à rédiger le texte de trois opéras français. L'administration du Théâtre devait se charger de la musique, peut-être Viotti lui-même d'ailleurs, ce qui n'aurait pas été sa première expérience en la matière. Pourtant jamais personne n'a trouvé la trace d'opéras créés par Collot d'Herbois. Le mot recoupe alors des réalités très diverses, néanmoins il s'agissait bel et bien pour lui d'une nouvelle expérience (11). L'un au moins de ces textes a-t-il survécu dans ses papiers? Le mystère reste encore entier et nous en sommes réduits à espérer que de nouveaux documents puissent émerger un jour futur. Pour l'heure, je me contenterai d'apprécier à sa juste valeur la découverte de ce manuscrit qui vient nous confirmer que l'abandon du théâtre par un homme qui lui avait consacré vingt ans de sa vie ne se fit de toute évidence pas dans la facilité.

Michel BIARD

(10) Le Thêâtre de Monsieur est l'un de ceux qui passaient pour être politiquement fort modérés : «Le théâtre de Monsieur est presque le seul où l'on n'ait jamais hasardé sur la scène de ces pièces incendiaires dont le moindre défaut est d'insulter lâchement et sans pudeur une classe de citoyens persécutés qu'il faut ramener par la douceur; on a joué chez Monsieur peu de pièces révolutionnaires et celles qu'on y représente tiennent si peu de la circonstance qu'elles feront le mème plaisir dans tous les temps [...]" (Almanach général des spectacles [...], 1792, op. cit.).

(11) Sauf si les administrateurs du Théâtre de la rue Feydeau utilisent ici le mot «opéra » pour désigner en fait de petites comédies entremêlées de vaudevilles. En ce cas, Collot d'Herbois a bien sûr déjà utilisé les vaudevilles. 


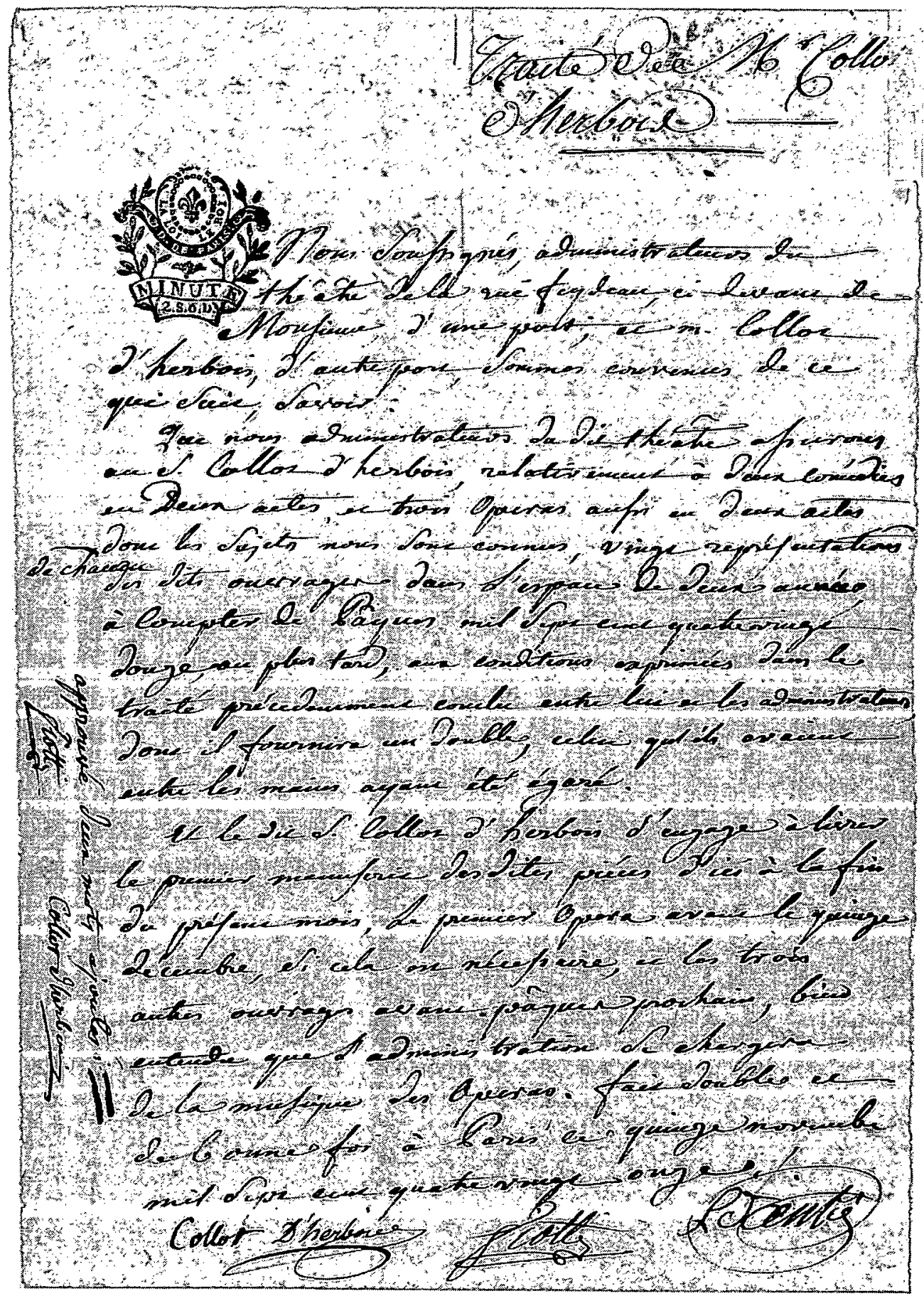

